\title{
Ataxia, CTCAE 5.0
}

National Cancer Institute

\section{Source}

National Cancer Institute. Ataxia, CT CAE 5.0. NCI Thesaurus. Code C146737.

A disorder characterized by lack of coordination of muscle movements resulting in the impairment or inability to perform voluntary activities. 